\title{
New molecular targets in bone metastases
}

\author{
D. Santini ${ }^{a}$, S. Galluzzo ${ }^{a}$, A. Zoccoli ${ }^{a}$, F. Pantano ${ }^{a}$, M.E. Fratto ${ }^{a}$, B. Vincenzi ${ }^{a}$, L. Lombardi ${ }^{b}$, C. Gucciardino ${ }^{a}$,

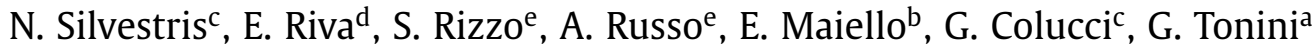 \\ ${ }^{a}$ Medical Oncology Dept, University Campus Bio-Medico, Rome, Italy \\ ${ }^{b}$ Medical Oncology Unit, IRCCS Casa Sollievo della Sofferenza, San Giovanni Rotondo, Foggia, Italy \\ ${ }^{c}$ Medical and Experimental Oncology Unit, Oncology Institute Giovanni Paolo II, Bari, Italy \\ ${ }^{d}$ Microbiology and Virology Dept, University Campus Bio-Medico, Rome, Italy \\ ${ }^{e}$ Section of Medical Oncology, Department of Surgical and Oncological Sciences, Università degli Studi di Palermo, Palermo, Italy
}

\section{A R T I C L E I N F O}

\section{Keywords:}

Molecular targets

Bone metastases

\section{S U M M A R Y}

Bone metastases have a major impact on morbidity and on mortality in cancer patients. Despite its clinical relevance, metastasis remains the most poorly elucidated aspect of carcinogenesis. The biological mechanisms leading to bone metastasis establishment have been referred as "vicious circle", a complex network between cancer cells and the bone microenvironment. This review is aimed to underline the new molecular targets in bone metastases management other than bisphosphonates. Different pathways or molecules such as RANK/RANKL/OPG, cathepsin K, endothelin-1, Wnt/DKK1, Src have recently emerged as potential targets and nowadays preclinical and clinical trials are underway. The results from those in the advanced clinical phases are encouraging and underlined the need to design large randomised clinical trials to validate these results in the next future.

Targeting the bone by preventing skeletal related events (SREs) and bone metastases has major clinical impact in improving survival in bone metastatic patients and in preventing disease relapse in adjuvant setting.

(C) 2010 Elsevier Ltd. All rights reserved.

\section{Introduction: Bone metastases - from the niche to "vicious circle"}

Bone metastases are the major cause of morbidity, and ultimately mortality, in cancer patients. ${ }^{1}$ Despite its clinical relevance, metastasis remains the most poorly elucidated aspect of carcinogenesis.

The establishment of bone metastasis is driven by a complex network between cancer cells and the bone microenvironment which is classically referred as "vicious circle". At the earliest steps, prior to the establishment of the vicious cycle, cancer cells and their associated stromal cells alter the marrow environment and prime it by secreting a magnitude of growth factors and cytokines. Bone marrow-derived hematopoietic progenitors that express vascular endothelial growth factor receptor 1 (VEGFR1+) mobilize in response to the unique pattern of growth factors and chemokines produced by the primary tumor. Their arrival in distant sites acts on the local microenvironment, termed the "premetastatic niche," which dictate the profile of metastatic spread. ${ }^{2}$

Importantly, examination of bone marrow metastases identified different determinants which might predict for later distant relapse.

\footnotetext{
* Corresponding author. Prof. Daniele Santini, Medical Oncology, University Campus Bio-Medico, Via Alvaro del Portillo, 200, 00128 Rome, Italy. Tel.: +39062 254112 06; fax: +39062 25411936 . E-mail address: d.santini@unicampus.it (D. Santini).
}

Identifying which of these cells have higher metastatic potential is of major clinical significance and therapeutic approach.

In order to form bone metastasis, cancer cells first have to metastasize to the bone marrow which is mainly composed of hematopoietic stem cells (HSCs) residing in two different biological structures known as osteoblastic and vascular niches.

The "osteoblastic niche" is mainly formed by osteoblasts lining at endosteal bone surface and providing a quiescent environment for HSCs maintenance and expansion into different hematopoietic lineages.

Whereas, the vascular niche, identified in association with fenestrated endothelium of sinusoids, drives HSCs transendothelial migration via endothelium-derived factors priming.

Communications between osteoblasts as well as other tumour stromal cells and HSCs are mainly driven via chemoattractive factors such as the stromal-derived factor 1 (SDF-1) on stromal cells and its receptor CXCR4 on HSCs. ${ }^{3}$

In addition, a cancer-related disruption of the homeostatic RANKRANKL loop between osteoclasts and osteoblasts occurs. Metastatic prostate carcinomas can secrete high amounts of the RANKL inhibitor osteoprotegerin, thereby attenuating osteoclastic reactions during metastasis. ${ }^{4}$ Conversely, osteolytic cancer cells can secrete proteases that cleave RANKL into a more active form, thereby activating the osteoclasts. ${ }^{5}$ 


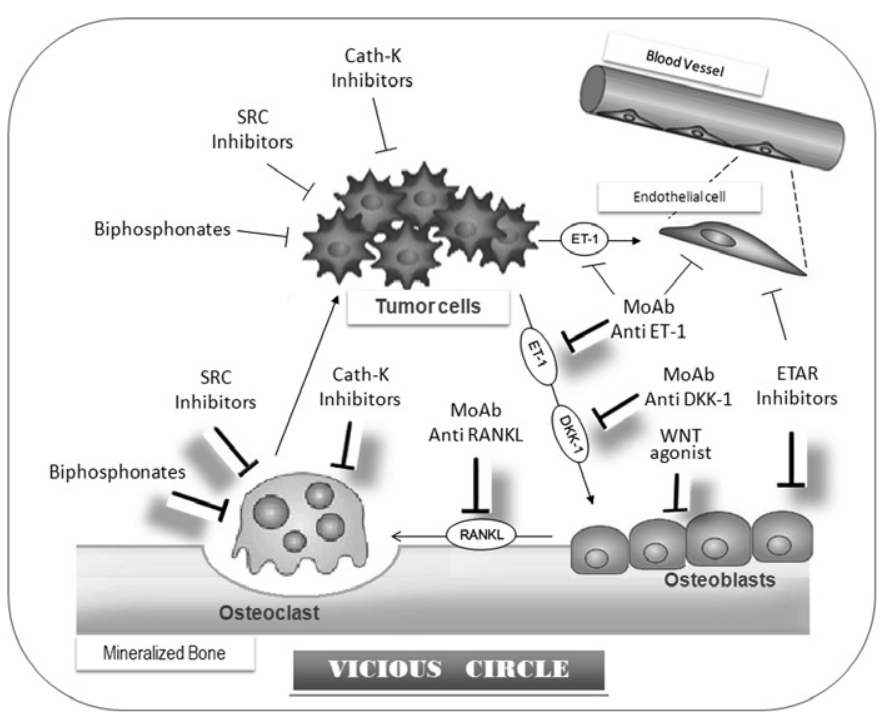

Fig. 1. Vicious circle pathways and/or molecules potential candidates in targeting bone metastases.

Moreover, because bone is a rich source of matrix-embedded growth factors such as IGF and TGF- $\beta$, these cytokines are released upon osteolysis and act upon the invading tumor cells to promote the induction of osteoclast-promoting factors. This positive feedback loop, referred to as "the vicious cycle of osteolytic bone metastasis," may indeed be the driving force for the selection of osteolytic mechanisms during the development of bone metastases. ${ }^{6}$

It is well known amino-bisphosphonates represent the standard treatment for bone metastasis. This class of compounds, and particularly zoledronic acid, has been shown to display antitumor effects, direct and indirect, by interfering with a variety of cells such as tumor cells, osteoclasts, HCSs, MDCS, gamma delta T cells and other immune cells and thus to inhibit the vicious cycle at different levels.

This review is aimed to summarized the new molecular targets in bone metastases management other than bisphosphonates. Different pathways or molecules such as RANK/RANKL, cathepsin K, endothelin 1, Wnt/DKK1, Src, etc have recently emerged as potential candidates in establishing and development of bone metastasis (Fig. 1). Nowadays preclinical and clinical trials are evaluating the possibility to therapeutically target these pathways.

\section{RANK/RANKL/OPG pathway}

Receptor Activator of nuclear Factor-kB Ligand (RANKL), the Receptor Activator of Nuclear Factor-kB (RANK) and the decoy Receptor Osteoprotegerin (OPG) are members of the TNF and TNFreceptor superfamily able to induce proliferation, differentiation, activation and apoptosis of osteoclastic cells. Bone remodelling is mediated by the interaction among RANKL expressed on the osteoblasts, RANK expressed on the osteoclast surface and OPG, the decoy receptor for RANKL that prevents osteoclast activation. Stromal osteoblast cells support osteoclast differentiation by their ability to secrete IL-6 and RANKL, in response to PTH, 1,25-hydroxyvitamin D3, and PGE2. ${ }^{7}$ The interaction between RANK/RANKL begins with the stimulation of RANK by RANKL promoting proliferation, differentiation and survival of osteoclast precursors and activation of mature osteoclasts. OPG, interacting with RANKL, counteracts these effects on osteoclasts.

Thus, RANKL/OPG levels determine bone resorption.

For this biological effect, RANK/RANKL/OPG pathway displays a key role in the growth of bone metastases: RANKL activates osteoclast-mediated bone resorption with a consequent release of matrix growth factors, like Tumor Growth Factor- $\beta$ (TGF- $\beta$ ) and platelet-derived growth factor (PDGF). The release of these factors can further induce the growth of tumor cells establishing a positive feedback mechanism. ${ }^{8}$ Moreover, cytokines, chemokines, growth factors and hormones derived from tumor cells upregulate RANKL through parathyroid hormone-related peptide (PTHrP), downregulating OPG. ${ }^{9}$ Increasing evidences show a direct role of RANK/RANKL interaction in bone metastases development ("vicious circle"). RANK not only is expressed on osteoclasts, but also on cancer cells. ${ }^{10}$ Preclinical evidences on animal models of osteolytic metastases reported targeting RANK/RANKL pathway lead to tumoral growth inhibition with osteolysis reduction.

\section{Denosumab}

Denosumab (AMG 162) is a noncytotoxic IgG2 monoclonal antibody with an extremely high affinity for human RANKL and a long halflife, which permits less frequent dosing. ${ }^{11}$ It was developed to treat patients with skeletal pathologies mediated by osteoclasts, such as bone metastasis, multiple myeloma, and cancer treatmentinduced bone loss (CTIBL). On the basis of preclinical evidences, many clinical trials were conducted to investigate denosumab in metastatic bone disease. Specifically, we are waiting for the final results of the three ongoing clinical trials evaluating denosumab versus ZA in metastatic breast cancer, prostate cancer as well as advanced solid tumors and multiple myeloma. Specifically at ECCO 2009 Stopeck et al. presented the first results regarding the Phase III study evaluating denosumab versus zoledronic acid (ZA) in breast cancer. They demonstrated that denosumab was superior to ZA and reduced the risk of a first on-study SRE by $18 \%$ (HR: $0.82,95 \% \mathrm{CI}$ : $0.71,0.95$; P value was less than 0.0001 for noninferiority and equal to 0.01 for superiority study) and of first and subsequent SRE by $23 \%$ on multiple event analysis (HR: $0.77 ; 95 \% \mathrm{CI}: 0.66,0.89 ; \mathrm{P}=0.001$ ). ${ }^{12}$ Also the Phase III study evaluating denosumab versus ZA in other solid tumors plus multiple myeloma showed denosumab was not inferior compared with ZA for delaying the time to the first onstudy SRE (HR: $0.84,95 \% \mathrm{CI}: 0.71,0.98$ ) (statistically significant for non inferiority $\mathrm{p}<0.0007$ ). The time to first SRE and the time to first-and-subsequent SRE was also numerically greater but not statistically superior compared to ZA (HR: 0.90, 95\% CI: 0.77, 1.04). ${ }^{13}$ At ASCO 2010 the results of the trial evaluating denosumab vs ZA in bone metastases from advanced cancer or multiple myeloma were presented. The time to first SRE or hypercalcemia of malignancy was significantly longer in the denosumab group (HR: 0.83 [95\% CI: $0.71,0.97], \mathrm{p}=0.02$ ) as was time to first radiation to bone (HR: 0.78 ; $95 \% \mathrm{CI}: 0.63,0.97, \mathrm{p}=0.03) .{ }^{14}$ At ASCO 2010 the study evaluating denosumab compared with ZA for the treatment of bone metastases in patients with castration-resistant prostate cancer was presented. Denosumab significantly delayed the time to first on-study SRE compared with ZA, (HR: 0.82; 95\% CI: 0.71, 0.95; p=0.008.) The median time to first on-study SRE was 20.7 mo denosumab vs. 17.1 mo ZA. Denosumab also significantly delayed the time to first and subsequent on-study SRE (multiple event analysis) (HR: 0.82; 95\% CI: 0.71, 0.94; p=0.004). ${ }^{15}$

\section{Endothelin pathway}

The Endothelins (ET) are peptides containing 21 amino acids produced by a variety of normal cells, such as endothelial cells, vascular smooth muscle cells and various epithelial tissues. The endothelin family comprises four isoforms ET-1-4 (the most recently identified). Presently, ET-1 is the most clinically relevant isoform. The conversion to the active ET-1 form, after proteolytic cleavage of its inactive precursor, is the main regulatory step in controlling ET-1 levels within the body. Endothelins exert their effects by binding to two distinct $G$ protein-coupled receptors, 
designed as ETAR and ETBR, with different affinity for the two receptors. Most importantly, the signaling events linked to ETRs activation induce divergent intracellular effects when mediated by the ETAR or ETBR activation. Moreover, different cell types associated within solid tumors (such as tumor cells, fibroblats, macrophage and endhotelial cells) overexpress ET1 and its receptors. ET- 1 exerts direct effects on cancer cells by acting through ETB and ETA receptors. Activation of ETB receptor by ET-1 induces apoptosis in human cancer cell lines while activation of ETA receptor drives cancer cell growth and proliferation. ET-1 exerts also indirect effects on tumor cells. In cancers, ET-1 exerts a tumorpromoting action through direct angiogenic effects on endothelial cells. ETA receptor expressed on endothelial cells and activated by ET-1 stimulates VEGF production by endothelial cells leading to increased tumor angiogenesis. ${ }^{16}$ ET-1 also stimulates osteoblasts proliferation in the bone marrow via MAPK phosphorylation and decreases osteoclastic bone resorption and motility.

In the bone microenvironment, ET-1 released from prostate bone metastases activates ETRA on osteoblasts leading to their proliferation and to bone density increase. Moreover, proliferating osteoblasts release growth factors which stimulate survival and growth of tumour cells in the bone microenvironment.

The involvement of ET1 in the hallmarks of cancer identify ET axis as a potential therapeutic target. Blocking the ETA receptor could inhibit osteoblastic bone growth and proliferation of metastases. Atrasentan is an inhibitor of the ET-A receptor that has been shown to block formation of osteoblastic metastases in mice. In a placebocontrolled phase II trial in men with asymptomatic hormonerefractory prostate metastatic cancer atrasentan significantly delayed the time to disease progression compared with placebo. ${ }^{17}$ In a subsequent placebo-controlled phase III trial in men with metastatic prostate cancer, atrasentan $(10 \mathrm{mg} /$ day $)$ did not reduce the risk of disease progression and cancer-induced bone pain neither an overall survival benefit was detected. ${ }^{18}$ Recently, a Phase 2 study to investigate the safety and efficacy of the specific ETAR antagonist ZD4054 in patients with metastatic hormoneresistant prostate cancer who were pain free or mildly symptomatic for pain was carried out. Patients were randomized into 3 groups to receive once-daily oral tablets of ZD4054 $10 \mathrm{mg}$, or $15 \mathrm{mg}$, or placebo. The primary end point of time to progression was not achieved in this study, but an improvement was seen in overall survival in both active treatment arms. ${ }^{19}$ Zibotentan (ZD4054) is an oral, specific ETA receptor antagonist. This drug is currently under clinical evaluation through a program consisting of three randomized double-blind trials called ENTHUSE (ENdoTHelin A USE) M0, M1 and M1c. ENTHUSE M0 (Study 0015, ClinicalTrials.gov identifier NCT00626548) is enrolling patients with HRPC with increasing PSA levels but no evidence of metastatic spread.

Patients will be accrued and randomized to zibotentan or placebo. Primary endpoints are overall survival, progression-free survival, defined as the time to appearance of metastases. Secondary endpoints include safety and tolerability, PSA levels and healthrelated quality of life. ENTHUSE M1 (Study 0014, ClinicalTrials.gov identifier NCT00554229), investigates zibotentan in HRPC patients with asymptomatic or mildly symptomatic metastases for whom chemotherapy is not yet appropriate randomized to zibotentan or placebo. The primary endpoint is overall survival; secondary endpoints include progression-free survival, safety and tolerability, skeletal events and bone metastases, PSA levels, and healthrelated quality of life. ENTHUSE M1c (Study 0033, ClinicalTrials.gov identifier NCT00617669) HRPC patients with confirmed symptomatic metastases will be randomized to receive zibotentan with docetaxel or docetaxel alone. The primary endpoint is overall survival. Secondary endpoints include progression-free survival, PSA levels, safety and tolerability, and the effect of treatment on skeletal events.

\section{Cathepsin K pathway}

Cathepsins are a class of globular lysosomal proteases that belong to the papain-like cysteine protease family. During the last 10 years Cathepsin K (CathK) has been the focus of increasing attention. It is expressed in a wide variety of tissues including the bone in which appears to be the most abundant and specific cysteine protease expressed by osteoclasts and osteoclast-like cells (giant multinucleated cells). Other cells within the bone milieu (e.g. osteoblasts and osteocytes) express cathepsin $\mathrm{K}$ although its role in these cells is not well known.

Cathepsin $\mathrm{K}$ represents the key enzyme responsible for osteoclastic bone resorption actively participating in the process of bone turnover. This cysteine protease plays a key role in bone matrix degradation and appears to be a limiting step in osteoclastic bone resorption. ${ }^{20}$

The well established role of cathK in bone resorption makes it an attractive therapeutic target in the treatment of those disorders involving bone loss, such as osteoporosis and bone metastasis.

Different cancers express cathepsin $\mathrm{K}$ including prostate and breast cancers both of which have a high tendency to metastasize to bone. ${ }^{21}$ Until now, a role for cathepsin $\mathrm{K}$ in bone metastasis has been mainly attributed to its ability to effectively degrade native collagen I, a process necessary for the expansion of tumour within the bone.

These observations suggest that inhibition of cathepsin $\mathrm{K}$ may disrupt two processes essential to the development of bone metastases: cancer cell invasion and osteoclast-mediated bone resorption. Experimental data on animal tumor models have shown inhibitors of cathepsin $\mathrm{K}$ effectively suppress bone resorption. ${ }^{22}$ From this evidence, several small-molecule inhibitors of cathepsin $\mathrm{K}$ have been developed and will enter soon phase 1 experimental validation.

In vivo proof of concept study designed for treatment of osteolysis and tumor growth in metastatic bone disease, cathepsin $\mathrm{K}$ inhibitor (L-006235) showed to reduce the size of osteolytic lesions by $66 \%$ when administered 18 days after tumor cell inoculation and 61\% when administrated at the same time as tumor cell inoculation. ${ }^{23}$

Moreover, sequential treatment L006235 (30 and 100) plus zoledronic acid $(12 \mathrm{ug} / \mathrm{kg}$ ) demonstrated additive efficacy in terms of protection from osteolysis and tumor volume.

Due to its selectivity, Odanacatib is the only cathepsin $\mathrm{k}$ inhibitor in clinical development. ${ }^{24} \mathrm{~A}$ Phase II controlled study on women with breast cancer metastatic to bone randomized to receive daily administration of odanacatib ( $5 \mathrm{mg}$ ) or a single $4 \mathrm{mg}$ IV dose of zoledronic acid showed bone remodeling markers reduction (urinary NTx) after 4 weeks treatment. ${ }^{25}$

\section{SRC pathway}

The proto-oncogene Src (encoded by the c-src gene) is a nonreceptor, membrane-associated tyrosine kinases that belongs to Src family kinases.

Src is widely express, the majority of cell types display low levels of it while some others such as mature osteoclasts express high levels of it.

Src displays multiple functions both in physiological and pathological processes such as cell proliferation, migration, differentiation, survival angiogenesis, tumorigenesis and inflammation. In osteoclasts, Src is activated following integrin binding after cells attach to bone matrix to start the resorptive process and also after RANKL binding to RANK. Src signaling is a key pathway during normal, healthy bone turnover and has also been shown to be essential for the normal organization of the osteoclasts cytoskeleton. ${ }^{26}$ With regard to bone remodeling Src coordinates both osteoclast and osteoblast activities: it positively regulates osteoclasts survival and resorbing activity, and conversely, Src 
may negatively regulate osteoblast maturation through inhibition of runt-related transcription factor 2 (Runx2) regulated genes. ${ }^{27}$ Thus, Src kinase is essential for osteoclast activation and osteoblast inhibition. During tumorigenesis Src aberrantly activate certain physiological processes thereby supporting tumor growth, metastatization and tissue invasion. Moreover, an increased expression and activity of Src have been described in a wide variety of tumor types including prostate, colon and breast cancer. ${ }^{28}$ The Src role in patients with metastatic bone disease is of particular importance given its involvement in both bone turnover and bone metastases formation. An increased Src-mediated osteoclastic activity leads to an uncontrolled release of growth factors (such as TGF- $\beta$, IGF I, II, PDGF) into the bone microenvironment due to the enhanced bone resorption process. Evidence for the importance of Src in the formation of bone metastases comes from studies in mice showing how Src inhibition or disruption results in a diminished capacity of bone metastases development.

Increased Src activity is known to correlate with tumor progression, with the highest activity in metastatic tissue. Preclinical studies have demonstrated that decreased c-Src expression enhances osteoblast proliferation and bone formation. ${ }^{29}$ Different Src inhibitors have been developed by the pharmaceutical industry with several of them in advanced clinical trials.

Saracatinib (AZD0530) is an orally active small-molecular-weight inhibitor of c-Src and BCR-Abl. Its efficacy in bone resorption has been demonstrated in two phase I clinical trials. ${ }^{30}$ Dasatinib, saracatinib, and bosutinib are currently being investigated in early clinical trials in patients with prostate or breast cancer. Results have also been reported from a phase $1 / 2$ study of dasatinib administered in combination with docetaxel in patients with progressive CRPC. Bone markers (uNTX, BAP) decrease, a PSA decline, and RECIST partial response was registered. ${ }^{31}$

In a phase 2 trial of saracatinib ( $175 \mathrm{mg}$ daily) in patients with advanced CRPC, treatment was well tolerated and five patients had a slight reduction in PSA. However, bone effects were not reported. ${ }^{32}$ In a small study of patients with multiple myeloma, dasatinib had effects on markers of osteoclast but not osteoblast function. ${ }^{33}$

Several clinical trials are ongoing with SRC inhibitors to evaluate bone markers as a specified endpoint in addition to tumor responses, including the randomized phase 3 trial of dasatinib in combination with docetaxel (NCT00744497). Trials in breast cancer include: two randomized phase 2 studies of the aromatase inhibitors exemestane (NCT00767520) or letrozole (NCT00696072) administered with or without dasatinib; a randomized phase 2 study of fulvestrant (estrogen receptor antagonist) with or without dasatinib (NCT00566618); a phase $1 / 2$ study of dasatinib in combination with zoledronic acid (NCT00566618); and a phase 2 of dasatinib administered either once or twice daily in patients with breast cancer and bone metastases (NCT00410813). Saracatinib effects on bone markers will be evaluated in a randomized phase 2 trial versus zoledronic acid in patients with prostate or breast cancer (NCT00558272) and a phase 2 study of patients with metastatic hormone receptor-negative or locally advanced unresectable breast cancer (NCT00559507). Randomized phase 2 trials of exemestane (NCT00793546) or letrozole (NCT00880009) with or without bosutinib are either planned or ongoing, respectively, although it is currently unclear if bone markers will be assessed.

\section{WNT pathway}

Wnt are cysteine-rich, secreted glycoproteins involved in embryonic development, tissue induction, and axial polarity. Wnt ligands activate several different receptor-mediated signal transduction pathways, together with that mediated by $\beta$-catenin, known as the canonical pathway. The Wnt pathway has been demonstrated to be a major signaling pathway in osteoblasts In the presence of Wnt ligand, Wnt binds to the receptor Fz and to the coreceptor LRP-5/6, leading to Dvl activation, which results in the disassociation of the multi-protein complex and the intracellular accumulation of $\beta$-catenin. Expression of Wnt10b in transgenic mice increased bone mass and overexpression of Wnt7b and $\beta$-catenin in C3H10T1/2 cells induced osteoblast differentiation. ${ }^{34}$ However, the exact mechanism by which Wnt signaling affects osteoblast differentiation remains to be elucidated.

The canonical Wnt pathway is regulated by a soluble antagonists that can be divided into two functional classes: dickkopf (DKK) family and secreted frizzled-related proteins (sFRP). Members of the DKK family bind to the LRP5/LRP6 component of the Wnt receptor complex, while sFRP bind to Wnt proteins: both result in a suppression of Wnt-signaling and a reduced osteoblast function. To date, four Dkk proteins have been identified in mammals ${ }^{35}$ among which Dkk1 and Dkk2 have been well characterized. DKK-1 has been shown to increase RANKL/OPG ratio. For this reason elevated DKK1 levels may enhance osteoclastogenesis. Wnt signaling in osteoblasts upregulates OPG expression and down-regulates RANKL expression, ${ }^{36}$ suggesting a mechanism by which Wnt signaling in osteoblasts indirectly regulates osteoclastogenesis. Data from several tumor phenotypes suggest that DKK1 promotes osteolytic metastases, and may facilitate the conversion of osteoblastic metastases to an osteolytic phenotype. Prostate cancers usually express lower DKK1 levels compared with normal prostate tissue. In prostate cancer cells DKK1 was found to block osteoblastic metastases without affecting tumor growth, while inhibition of DKK1 in osteolytic prostate cancer cells switched bone metastases from osteolytic to osteoblastic Anti-DKK-1 therapy on bone metabolism and tumor growth was recently experimented in mice: DKK-1-neutralizing antibodies restored the BMD of the implanted myelomatous bone, increased the numbers of osteocalcin-expressing osteoblasts and reduced number of multinucleated TRAP-expressing osteoclasts. Furthermore, the anti-DKK-1-treated mice showed reduced tumor burden. ${ }^{37}$ BHQ880, a fully human anti-DKK-1 neutralizing antibody, promotes bone formation and inhibits tumor-induced osteolytic disease in preclinical studies. ${ }^{38}$

Taken together, these studies indicate WNT/DKK1 pathway is a key regulator of bone remodelling in physiologic and pathologic conditions. Target this pathway may potentially result in stimulation of osteoblastogenesis and inhibition of osteoclastogenesis in solid tumours.

\section{Conclusion: Potential new targets and future perspective}

In summary, a variety of novel targets in management of bone disease are underway in preclinical and clinical testing phases. The results from those in the advanced clinical phases are encouraging and underlined the need to design large randomised clinical trials to validate these results in the next future. Denosumab is the molecule which is in the most advanced development phase, presently being tested in Phase III trials. Further definition of the molecular and cellular pathways involved into bone metastasis biology is ongoing and it will likely lead to new and improved therapeutic strategies in bone metastasis. For example Notch is emerged as an important target in bone homeostasis. Mice knocked down for Notch lost the ability to suppress bone resorption. Targeting Notch pathway by gamma secretase inhibitors could represent in the next future a feasible therapeutic strategy.

Moreover, many new therapeutic targets in bone metastases, including TGF- $\beta$, Src, CXCR4, GPNMB and EGF-family ligands are currently under pre clinical investigation. 
Targeting the bone by preventing skeletal related events (SREs) and bone metastases has major clinical impact in improving survival in bone metastatic patients and in preventing disease relapse in adjuvant setting.

\section{Acknowledgements}

On behalf of Italian Society of Osteoncology.

\section{Conflict of interests}

All authors declare no conflict of interest.

\section{References}

1. Coleman RE. Metastatic bone disease: clinical features, pathophysiology and treatment strategies. Cancer Treat Rev 2001;27(3):165-76.

2. Kaplan RN, Rafii S, Lyden D. Preparing the "soil": the premetastatic niche. Cancer Res 2006;66(23):11089-93.

3. Jung Y, Wang J, Schneider A, et al. Regulation of SDF-1 (CXCL12) production by osteoblasts; a possible mechanism for stem cell homing. Bone 2006;38(4):497508

4. Logothetis CJ, Lin SH. Osteoblasts in prostate cancer metastasis to bone. Nat Rev Cancer 2005;5:21-8.

5. Lynch CC, Hikosaka A, Acuff HB, et al. MMP-7 promotes prostate cancer-induced osteolysis via the solubilization of RANKL. Cancer Cell 2005;7:485-96.

6. Kang Y, Siegel PM, Shu W, et al. A multigenic program mediating breast cancer metastasis to bone. Cancer Cell 2003:3:537-49.

7. Liu XH, Kirschenbaum A, Yao S, Levine AC. Cross-talk between the interleukin-6 and prostaglandin $\mathrm{E}(2)$ signaling systems results in enhancement of osteoclastogenesis through effects on the osteoprotegerin/receptor activator of nuclear factor-\{kappa\}B (RANK) ligand/RANK system. Endocrinology 2005; 146(4):1991-8.

8. Uehara H, Kim SJ, Karashima T, et al. Effects of blocking platelet-derived growth factor-receptor signaling in a mouse model of experimental prostate cancer bone metastases. J Natl Cancer Inst 2003;95(6):458-70.

9. Guise TA. Molecular mechanisms of osteolytic bone metastases. Cancer 2000;88(12 Suppl):2892-8

10. Mori K, Le Goff B, Charrier C, Battaglia S, Heymann D, Rédini F. DU145 human prostate cancer cells express functional receptor activator of NFkappaB: new insights in the prostate cancer bone metastasis process. Bone 2007:40:981-90.

11. Kostenuik PJ. Osteoprotegerin and RANKL regulate bone resorption, density, geometry and strength. Curr Opin Pharmacol 2005;5:618-25.

12. Stopeck A, Body JJ, Fujiwara Y, et al. Denosumab versus zoledronic acid for the treatment of breast cancer patients with bone metastases: results of a randomized phase 3 study. Eur J Cancer Suppl 2009;7(3):2-3.

13. Henry D, von Moos R, Vadhan-Raj S, et al. A double-blind, randomized study of denosumab versus zoledronic acid for the treatment of bone metastases in patients with advanced cancer (excluding breast and prostate cancer) or multiple myeloma. Eur J Cancer Suppl 2009;7:11-2.

14. Vadhan-Raj S, Henry DH, von Moos R, et al. Denosumab in the treatment of bone metastases from advanced cancer or multiple myeloma (MM): Analyses from a phase III randomized trial. J Clin Oncol 2010;28(Suppl):15s [abstract 9042].

15. Fizazi K, Carducci MA, Smith MR, et al. A randomized phase III trial of denosumab versus zoledronic acid in patients with bone metastases from castration-resistant prostate cancer. J Clin Oncol 2010;28(Suppl):18s [abstract LBA4507].

16. Bagnato A, Spinella F. Emerging role of endothelin-1 in tumor angiogenesis. Trends Endocrinol Metab 2003;14(1):44-50.

17. Carducci MA, Padley RJ, Breul J, et al. Effect of endothelin-A receptor blockade with atrasentan on tumor progression in men with hormone-refractory prostate cancer: a randomized, phase II, placebo-controlled trial. J Clin Oncol 2003;21(4): 679-89.

18. Carducci MA, Saad F, Abrahamsson PA, et al. Atrasentan Phase III Study Group Institutions. A phase 3 randomized controlled trial of the efficacy and safety of atrasentan in men with metastatic hormone-refractory prostate cancer. Cancer 2007;110(9):1959-66.

19. James ND, Caty A, Borre M, et al. Safety and efficacy of the specific endothelin-A receptor antagonist ZD4054 in patients with hormone-resistant prostate cancer and bone metastases who were pain free or mildly symptomatic: a double-blind placebo-controlled, randomised, phase 2 trial. Eur Urol 2009;55(5):1112-23.

20. Garnero P, Borel O, Byrjalsen I, et al. The collagenolytic activity of cathepsin $\mathrm{K}$ is unique among mammalian proteinases. J Biol Chem 1998;273(48):32347-52.

21. Brubaker KD, Vessella RL, True LD, Thomas R, Corey E. Cathepsin K mRNA and protein expression in prostate cancer progression. J Bone Miner Res 2003;18(2): 222-30.

22. Votta BJ, Levy MA, Badger A, et al. Peptide aldehyde inhibitors of cathepsin K inhibit bone resorption both in vitro and in vivo. J Bone Miner Res 1997;12(9): 1396-406.

23. Le Gall C, Bellahcène A, Bonnelye $\mathrm{E}$, et al. A Cathepsin $\mathrm{K}$ inhibitor reduces breast cancer induced osteolysis and skeletal tumor burden. Cancer Res 2007;67(20): 9894-902.

24. Gauthier JY, Chauret N, Cromlish W, et al. The discovery of odanacatib (MK-0822), a selective inhibitor of cathepsin K. Bioorg Med Chem Lett 2008;18(3):923-8.

25. Jensen AB, Olmeo N, Wynne C Ramirez G, et al. Effect of cathepsin K inhibition on suppression of bone resorption in women with breast cancer and established bone metastases in a 4-week, double-blind, randomized controlled trial. J Clin Oncol 2008;26(May 20 Suppl): Abstr. 1023.

26. Miyazaki T, Sanjay A, Neff L, Tanaka S, Horne WC, Baron R. Src kinase activity is essential for osteoclast function. J Biol Chem 2004;279:17660-6.

27. Zaidi SK, Sullivan AJ, Medina R, et al. Tyrosine phosphorylation controls Runx2 mediated subnuclear targeting of YAP to repress transcription. Embo J 2004;23: 790-9.

28. Frame MC. Src in cancer: deregulation and consequences for cell behaviour. Biochim Biophys Acta 2002;1602:114-30.

29. Marzia M, Sims NA, Voit S, et al. Decreased c-Src expression enhances osteoblast differentiation and bone formation. J Cell Biol 2000;151(2):311-20.

30. Rucci N, Susa M, Teti A. Inhibition of protein kinase c-Src as a therapeutic approach for cancer and bone metastases. Anticancer Agents Med Chem 2008;8(3):342-9.

31. Araujo J, Armstrong AJ, Braud EL, et al. Dasatinib and docetaxel combination treatment for patients with castration-resistance progressive prostate cancer: A phase I/II study (CA180086). J Clin Oncol 27(15S):249s (abstr 5061).

32. Lara Jr PN, Longmate J, Evans CP, et al. A phase II trial of the Src-kinase inhibitor AZD0530 in patients with advanced castration-resistant prostate cancer: a California Cancer Consortium study. Anticancer Drugs 2009;20(3):179-84.

33. Wildes TM, Procknow E, Weilbaecher K, Vij R. Effect of dasatinib on bone metabolism in multiple myeloma. J Clin Oncol 2008;26(May 20 Suppl): Abstr. 8568.

34. Hu H, Hilton MJ, Tu X, Yu K, Ornitz DM, Long F. Sequential roles of Hedgehog and Wnt signaling in osteoblast development. Development 2005;132(1):49-60.

35. Kawano Y, Kypta R. Secreted antagonists of the Wnt signalling pathway. J Cell Sci 2003;116:2627-34.

36. Spencer GJ, Utting JC, Etheridge SL, Arnett TR, Genever PG. Wnt signalling in osteoblasts regulates expression of the receptor activator of NFkappaB ligand and inhibits osteoclastogenesis in vitro. J Cell Sci 2006;119:1283-96.

37. Yaccoby S, Ling W, Zhan F, Walker R, Barlogie B, Shaughnessy Jr JD. Antibodybased inhibition of DKK1 suppresses tumor-induced bone resorption and multiple myeloma growth in vivo. Blood 2007;109(5):2106-11.

38. Fulciniti M, Tassone P, Hideshima T, et al. Anti-DKK1 mAb (BHQ880) as a potential therapeutic agent for multiple myeloma. Blood 2009;114(2):371-9. 